\title{
Carbapenem and colistin resistance in children with Enterobacteriaceae infections
}

\author{
Zeliha Haytoğlu ${ }^{1 \oplus}$, Özlem Özgür Gündeşlioğlu ${ }^{2 \oplus}$, Dinçer Yıldızdaş ${ }^{3 \oplus}$, \\ Emine Kocabaşs ${ }^{2 \oplus}$, Derya Alabaz ${ }^{2 \oplus}$, Özden Özgür Horoz ${ }^{3 \oplus}$ \\ Divisions of ${ }^{2}$ Pediatric Infectious Diseases and ${ }^{3}$ Pediatric Critical Care, ${ }^{1}$ Department of Pediatrics, Çukurova University Faculty of \\ Medicine, Adana, Turkey.
}

\begin{abstract}
Background. Carbapenem-resistant Enterobacteriaceae (CRE) are an emerging global public health threat. As a reserve agent, colistin has been the drug of choice for the treatment of infections caused by CRE. The aim of this study was to determine the risk factors of carbapenem and colistin-resistant Enterobacteriaceae infections and to investigate the outcomes.
\end{abstract}

Methods. We conducted a retrospective study in a single university hospital between the years 2013 and 2017 including 150 patients with Enterobacteriaceae infections.

Results. Of 150 Enterobacteriaceae infections, 62 (41\%) were carbapenem and 23 (15\%) were colistin-resistant. Colistin resistance rates among Enterobacteriaceae species increased from 4\% in 2014 to $25 \%$ in 2017 . The inhospital mortality of the patients with colistin-resistant and with carbapenem-resistant infections were 39\% (9/23) and 45\% (28/62), respectively. Prior exposure to polyantibiotic therapy for Gram negative bacteria was found as a predictor of CRE $(\mathrm{OR}=6.4 ; 95 \% \mathrm{CI} 3.07-13.6 ; \mathrm{p}=0.001)$ infections. The median length of hospital stay prior to positive culture $(\mathrm{OR}=1.02 ; 95 \% \mathrm{CI}, 1.0-1.04 ; \mathrm{p}=0.003)$ and history of surgery during the admission $(\mathrm{OR}=2.46 ; 95 \% \mathrm{CI} 1.2-5.1 ; \mathrm{p}=0.005)$ were found as the predictors of CRE infections. Underlying necrotizing enterocolitis and/or short-bowel syndrome $(\mathrm{OR}=6.38 ; 95 \% \mathrm{CI} 1.16-35 ; \mathrm{p}=0.033)$ and mechanical ventilation prior to index culture were found as predictors of colistin resistance $(\mathrm{OR}=9.4 ; 95 \% \mathrm{CI} 2-40.4 ; \mathrm{p}=0.004)$.

Conclusions. Recognizing the risk factors of carbapenem and colistin resistant Enterobacteriaceae infections is essential in order to conserve carbapenem and colistin since there are no new antibiotics to treat multidrugresistant Enterobacteriaceae infections.

Key words: carbapenem-resistance, colistin-resistance, Enterobacteriaceae, outcome, risk factors.

Enterobacteriaceae species are common cause of infections in both community and healthcare settings worldwide. Carbapenems are the mainstay of therapy for infections caused by Enterobacteriaceae producing extendedspectrum beta-lactamases (ESBLs). The emergence and dissemination of carbapenem resistance among Enterobacteriaceae in all over the world represents a serious threat to public

\footnotetext{
Zeliha Haytoğlu

zelihahayt@yahoo.com
}

Received 15th October 2018, revised 26th May 2019, 21st June 2019, 6th August 2019, 3rd January 2020, accepted 6th January 2020. health and significantly limits the treatment options for life threatening infections. ${ }^{1}$ Although colistin is currently considered as a last-resort treatment for infections caused by multi-drug resistant (MDR) bacteria, colistin resistance in Enterobacteriaceae species has been reported in several countries around the world and its prevalence has continued to increase, thus becoming a great healthcare concern..$^{2-5}$ It is important for healthcare facilities to understand how common CRE are in their institutions. Recognizing the risk factors of carbapenem and colistin-resistant Enterobacteriaceae infections is essential in order to conserve carbapenem and colistin, since there are no new antibiotics to treat 
MDR Enterobacteriaceae infections. Interventions to control CRE are evolving as more data and experience become available. Although several factors that increase the risk of infection with CRE have been reported, there is limited data on the epidemiology, risk factors, treatment, and outcomes in pediatric populations with colistin-resistant Enterobacteriaceae infections. ${ }^{6-8}$

In this study it was aimed to characterize the clinical features of patients with carbapenem and colistin-resistant Enterobacteriaceae infections. The other goals were to identify the risk factors, investigate the outcomes and determine the frequency of colistin and carbapenem resistance in clinical isolates of Enterobacteriaceae species.

\section{Material and Methods}

The clinical and microbiological data of all patients with isolation of Enterobacteriaceae from different specimens between December 2013 and December 2017 were retrospectively evaluated in our university hospital. Only patients determined to have an active infection were included in the study. Patient demographics, comorbidities, dates of admission, outcomes, medications, history of surgery, use of mechanical ventilation, and procedures applied during the hospitalization were included in the study. To identify CRE definition CDC surveillance reports in 2015 were used. According to CDC reports CRE was identified in two situations; 1-Resistant to any carbapenem antimicrobial (i.e. minimum inhibitory concentrations of $\geq 4 \mathrm{mcg} / \mathrm{ml}$ for doripenem, meropenem, or imipenem or $\geq 2$ $\mathrm{mcg} / \mathrm{ml}$ for ertapenem) 2- For bacteria that have intrinsic imipenem non-susceptibility (i.e. Morganella morganii, Proteus spp., Providencia spp.), resistance to carbapenems other than imipenem. ${ }^{9}$ Identification and susceptibility testing of clinical isolates were performed using automated laboratory system; Vitek-2 (bioMérieux). Minimum inhibitory concentrations (MICs) were interpreted according to European Committee on
Antimicrobial Susceptibility testing (EUCAST) breakpoints. ${ }^{10}$ Empiric antimicrobial therapy was identified as the antibiotics those used before the index blood culture results and those used at least 48 hours. Healthcare-associated infections were diagnosed according to Centers for Disease Control and Prevention definitions. ${ }^{11}$ This study was approved by the Institutional Ethics Committee (5th October 2018; report number 81/5). All procedures performed in our study involving human participants were in accordance with the ethical standards of the institutional research committee and with the 1964 Helsinki declaration and its later amendments.

\section{Statistical analysis}

An $X^{2}$ test or Fisher exact test was used to compare the categorical variables, while Student t-test or Mann-Whitney U test was used to compare the continuous variables, as appropriate. Univariate and multivariate logistic regression analyses were used to assess factors associated with in-hospital mortality and CRE infections. In-hospital mortality was compared between the CRE group and CSE group by using Kaplan-Meier estimation and compared using the long-rank test. A p-value $<0.05$ was considered statistically significant for all tests. All analyses were performed with SPSS Statistics (version 21.0. IBM Corp, Armonk, New York).

\section{Results}

\section{Demographics and clinical history of the patients with CRE}

One-hundred-fifty patients with Enterobacteriaceae infections were included in the study; 76 were male $(50.7 \%), 74$ were female (49.3\%). In total 62 patients $(41.3 \%)$ were infected with CRE. Twenty-eight (45.2\%) of the CRE isolates were in an ICU ward (neonatal and pediatric). The wards in which patients with CRE infections were isolated are shown in Table I. 
Table I. Hospital location of patients with Enterobactericeae.

\begin{tabular}{lccc}
\hline Department & CRE $(\mathrm{n}=62)$ & CSE $(\mathrm{n}=88)$ & Total $(\mathrm{n}=150)$ \\
\hline Pediatric intensive care unit, $\mathrm{n}(\%)$ & $14(22.6)$ & $17(19.3)$ & $31(20.7)$ \\
Neonatal intensive care unit, $\mathrm{n}(\%)$ & $14(22.6)$ & $48(54.5)$ & $62(41.3)$ \\
Pediatric hematology and oncology, $\mathrm{n}(\%)$ & $15(24.2)$ & $17(19.3)$ & $32(21.3)$ \\
Pediatric cardiovascular surgery, $\mathrm{n}(\%)$ & $7(11.3)$ & $2(2.3)$ & $9(6)$ \\
Burn unit, $\mathrm{n}(\%)$ & $2(3.2)$ & $0(0)$ & $2(1.3)$ \\
Pediatric surgery, $\mathrm{n}(\%)$ & $1(1.6)$ & $3(3.4)$ & $2(1.3)$ \\
Other pediatric wards, $\mathrm{n}(\%)$ & $9(14.5)$ & $12(8)$ \\
\hline
\end{tabular}

CRE: carbapenem-resistant Enterobactericeae, CSE: carbapenem-sensitive Enterobactericeae

Carbapenem resistance was observed in 47 of 98 (47.9\%) Klebsiella species, in eight of 23 (34.7\%) E.coli species, in three of ten (30\%) Enterobacter species, in one of two (50\%) Citrobacter species and in three of eight (37.5\%) Stenotrophomonas species. CRE was isolated from blood in 51 $(82.3 \%)$ patients, from respiratory tract in eight $(12.9 \%)$, from urine in one patient $(1.6 \%)$ and from wound specimens in two patients (3.2\%). Ventilator-associated pneumonia (VAP) was observed in 22 patients (35.5\%) and pneumonia other than VAP was observed in six patients $(9.7 \%)$.

All patients were hospitalized for $>48$ hours before their CRE infection. Underlying medical conditions were reported in $54(87.1 \%)$ of 62 patients with CRE infection. The most common condition was cardiac disease (17 patients, $27.4 \%), 14(22.5 \%)$ had oncologic processes, nine $(14.5 \%)$ had pulmonary disease, nine $(14.5 \%)$ had a history of prematurity, four (6.4\%) patients had necrotizing enterocolitis and/or short-bowel syndrome. A history of surgery was reported in 31 (50\%) of 62 patients; 15 (48.3\%) of the procedures were gastrointestinal, and 10 $(32.2 \%)$ were cardiac procedures. An indwelling device was reported in $60(96.8 \%)$ of 62 children. Of 62 patients with CRE infections, bacteremia was observed in 51 patients $(82.2 \%)$. There was no statistical difference in terms of gender between CRE and carbapenem-susceptible Enterobacteriaceae (CSE) groups $(\mathrm{p}=0.25)$. The median age of patients in CRE group was 29 days (range: 4 days -2.71 years) and in the CSE group was 1.03 year (range: 5 days - 22.7 years). Patients in the CRE group were younger than the patients in the CSE group $(p=0.004)$. Demographics and clinical findings of patients with CRE are shown in Table II.

\section{Risk factors of CRE infections}

Although patients with CRE infections were younger and had higher rates of central catheter compared to the CSE group, in regression analysis neither age nor central line was associated with the risk of CRE infections. Risk factors of CRE infections are demonstrated in Table III. Prior to the index culture, empiric polyantibiotic therapy for Gram negative bacteria was detected in 31 (50\%) patients with CRE vs. 16 (18.2\%) patients with CSE. Prior exposure to polyantibiotic therapy for Gram negative bacteria was found as a risk factor of CRE $(p=0.001)$. The median length of hospital stay prior to positive cultures with CRE infections was longer than the length of hospital stay in patients with CSE infections $(p=0.003)$.

Logistic regression analysis revealed that three variables were independently associated with the isolation of CRE strains: length of hospitalization prior to index culture (OR, 1.02; 95\% CI, 1.01 -1.04, $\mathrm{p}=0.003)$, previous polyantibiotic therapy (OR, 6.4; 95\% CI, 3.07-13.6, $\mathrm{p}=0.001$ ) and history of surgical intervention during admission (OR, 2.46; 95\% CI, 1.2-5.1, $\mathrm{p}=0.005$ ). 
Table II. Demographics and clinical findings of patients with and without carbapenem-resistant Enterobacteriaceae.

\begin{tabular}{|c|c|c|c|}
\hline Demographics and clinical findings & CRE $(n=62)$ & CSE $(n=88)$ & $\mathrm{p}$ \\
\hline Age, median (range) & $\begin{array}{c}29 \text { days } \\
\text { (4 days }-2.7 \text { years) }\end{array}$ & $\begin{array}{c}1.03 \text { year } \\
\text { (5 days }-22.7 \text { years })\end{array}$ & $0.004^{*}$ \\
\hline Male, n (\%) & $28(45.1)$ & $48(54.5)$ & 0.25 \\
\hline Mechanical ventilation prior to index culture, $\mathrm{n}(\%)$ & $36(58)$ & $53(60.2)$ & 0.79 \\
\hline Central venous catheter prior to index culture, $\mathrm{n}(\%)$ & $60(96.8)$ & $77(87.5)$ & $0.047^{*}$ \\
\hline \multicolumn{4}{|l|}{ Empiric antibiotic exposure prior to index culture, $\mathrm{n}(\%)$} \\
\hline - Piperacillin/tazobactam & $4(6.4)$ & $4(4.5)$ & 0.39 \\
\hline - Carbapenem & $12(19.3)$ & $19(21.6)$ & 0.51 \\
\hline - 3rd or 4th generation cephalosporins & $4(6.5)$ & $13(14.8)$ & 0.87 \\
\hline - Aminoglycoside & $6(9.7)$ & $28(31.8)$ & 0.75 \\
\hline - Flouroquinolone & $4(6.5)$ & $3(3.4)$ & 0.21 \\
\hline - Carbapenem + aminoglycoside & $12(19.3)$ & $4(4.5)$ & $0.001^{*}$ \\
\hline - Carbapenem + except aminoglycoside & $8(12.9)$ & $7(7.9)$ & 0.072 \\
\hline - Aminoglycoside + except carbapenem & $11(17.7)$ & $5(5.7)$ & $0.004^{*}$ \\
\hline - No empiric therapy & $1(1.6)$ & $5(5.7)$ & \\
\hline $\begin{array}{l}\text { Length of hospital stay prior to index culture (days), } \\
\text { median (IQR); [min-max] }\end{array}$ & $26(12-42.5) ;[1-150]$ & 18 (12-31.5); [4-100] & \\
\hline Operation: yes, n (\%) & $31(50)$ & $26(29.5)$ & 0.015 \\
\hline
\end{tabular}

Table III. Predictors of carbapenem-resistant Enterobacteriaceae and colistin-resistant Enterobacteriaceae.

\begin{tabular}{lcc}
\hline Predictors for resistance & OR (95\% CI) & $\mathrm{P}$ \\
\hline Carbapenem resistance & & \\
$\quad$ - Length of hospitalization prior to index culture & $1.02(1.01-1.04)$ & $0.003^{*}$ \\
- Polyantibiotic therapy prior to index culture & $6.4(3.07-13.6)$ & $0.001^{*}$ \\
$\quad$ - History of surgical intervention during admission & $2.46(1.2-5.1)$ & $0.005^{*}$ \\
Colistin resistance & & \\
$\quad$ - Urinary catheter & $0.32(0.18-0.38)$ & $0.012^{*}$ \\
- Mechanical ventilation prior to index culture & $9.4(2.0-40.4)$ & $0.004^{*}$ \\
- Underlying necrotizing enterocolitis and/or short-bowel syndrome & $6.38(1.16-35)$ & $0.033^{*}$ \\
\hline$*$ * $<0.05$ & &
\end{tabular}

\section{Demographicsand clinicalhistoryofthepatients with colistin-resistant Enterobacteriaceae}

Twenty-three patients had colistin-resistant Enterobacteriaceae infections. The median age of the children with colistin-resistant Enterobacteriaceae infections was 150 days (range, 5 days -11.8 years). Nine (39\%) patients were male and $14(61 \%)$ patients were female. The median length of hospital stay prior to positive culture results of colistin-resistant species was 26 days (range, 5-150 days; interquartile range, 11-46 days). The rate of colistin resistance in Enterobacteriaceae species increased markedly from $4.9 \%$ in 2014 to $25 \%$ in 2017. In 2014 two out of 41 Enterobacteriaceae infections were colistin-resistant (4.9\%). In 2015 eight out of 37 infections (17.8\%), in 2016 six $(18.2 \%)$ were colistin-resistant and in 2017 seven (25\%) were colistin-resistant. Nineteen (82\%) patients were in an ICU ward (neonatal and pediatric). Underlying medical conditions were 
reported in all patients with colistin-resistant Enterobacteriaceae infections. The most common condition was cardiac disease (nine patients, $39 \%)$, six (26\%) had pulmonary disease, five $(21.7 \%)$ had a history of prematurity, three patients $(13 \%)$ had necrotizing enterocolitis and/or short-bowel syndrome. A history of surgery was reported in $11(47.8 \%)$ out of 23 patients. Central line was reported in 22 (95.7\%) patients. Mechanical ventilation prior to index culture was reported in 21 (91.3\%) patients. Urinary catheter was reported in 15 (65.2\%) patients. Source of isolates were blood in 20 $(87 \%)$ patients. Sixteen $(69.6 \%)$ colistin-resistant strains were Klebsiella species, six (26.1\%) were Serratia species, one (4.3\%) was E.coli species. Demographics and clinical findings of patients with CRE are shown in Table IV.

\section{Risk factors of colistin-resistant Enterobacteriaceae infections}

Mechanical ventilation prior to index culture were more common in patients with colistinresistant group (21/23) than colistin-susceptible group (68/127) which was $91.3 \%$ vs. $53.5 \%$ respectively. Risk factors of colistin resistance are shown in Table III. In the regression analysis mechanical ventilation prior to index culture was determined as a risk factor of colistin resistance (OR, 9.4; 95\% CI, 2-40.4; $\mathrm{p}=0.004)$.

Urinary catheter was reported higher in the colistin-resistant group (15/23) than the colistin sensitive group (46/127) which was $65.2 \%$ vs. $36.2 \%$, respectively. In logistic regression analysis urinary catheter was the risk factor of colistin resistance (OR, 0.32; 95\% CI, 0.18-0.38; $p=0.012$ ). The other independent risk factor of colistin resistance was underlying necrotizing enterocolitis and/or short-bowel syndrome (OR, 6.38; 95\% CI, 1.16-35; $\mathrm{p}=0.033$ ).

\section{Outcome in CRE and colistin-resistant Enterobacteriaceae infections}

The in-hospital mortality of the CRE group was $(28 / 62) 45.2 \%$. There were no statistically significant difference in mortality rates between CRE and CSE groups which was $45.2 \%$ vs. $36.7 \%(p=0.071)$. The median length of hospital stay after the index culture in CRE group was 21 days (range, 0-96 days) vs. 19 days (range, 0-160 days) in CSE group ( $p=0.12$ ) (Fig. 1). The inhospital mortality rates of patients infected by colistin-susceptible Enterobacteriaceae was 36.2\% (46/127) and colistin-resistant Enterobacteriaceae was 39.1\% (9/23). In Kaplan-Meier analysis there were no statistically significant difference

Table IV. Demographics and clinical findings of patients with and without colistin resistance Enterobacteriaceae.

\begin{tabular}{|c|c|c|c|}
\hline Demographics and clinical findings & $\begin{array}{l}\text { Colistin resistant } \\
\qquad(\mathrm{n}=23)\end{array}$ & $\begin{array}{l}\text { Colistin sensitive } \\
\qquad(\mathrm{n}=127)\end{array}$ & $\mathrm{P}$ \\
\hline Age, median (range) & $\begin{array}{c}150 \text { days } \\
\text { (5 days }-11.8 \text { years) }\end{array}$ & $\begin{array}{c}165 \text { days } \\
\text { (4 days }-22.7 \text { years) }\end{array}$ & 0.56 \\
\hline Male, n (\%) & $9(39.1)$ & $67(52.8)$ & 0.22 \\
\hline Urinary catheter, n (\%) & $15(65.2)$ & $46(36.2)$ & $0.009^{*}$ \\
\hline Underlying necrotizing enterocolitis, n (\%) & $3(13.1)$ & $4(3.1)$ & $0.038^{*}$ \\
\hline Mechanical ventilation prior to index culture, $\mathrm{n}(\%)$ & $21(91.3)$ & $68(53.5)$ & $0.001^{*}$ \\
\hline Piperacilin/tazobactam, n (\%) & $1(4.3)$ & $7(5.5)$ & 1.0 \\
\hline Carbapenem, n (\%) & $5(21.7)$ & $26(20.5)$ & 1.0 \\
\hline $3^{\text {rd }}$ or $4^{\text {th }}$ generation cephalosporin, n ( $\left.\%\right)$ & $2(8.7)$ & $15(11.8)$ & 0.66 \\
\hline Aminoglycoside, n (\%) & $3(13)$ & $31(24.4)$ & 0.23 \\
\hline Carbapenem + aminoglycoside, n (\%) & $3(13)$ & $13(10.2)$ & 0.36 \\
\hline Carbapenem + except aminoglycoside (\%) & $4(17.4)$ & $11(8.7)$ & 0.74 \\
\hline Aminoglycoside + except carbapenem, n (\%) & $5(21.7)$ & $11(8.7)$ & $0.035^{*}$ \\
\hline
\end{tabular}

*: $\mathrm{p}<0.05$ 


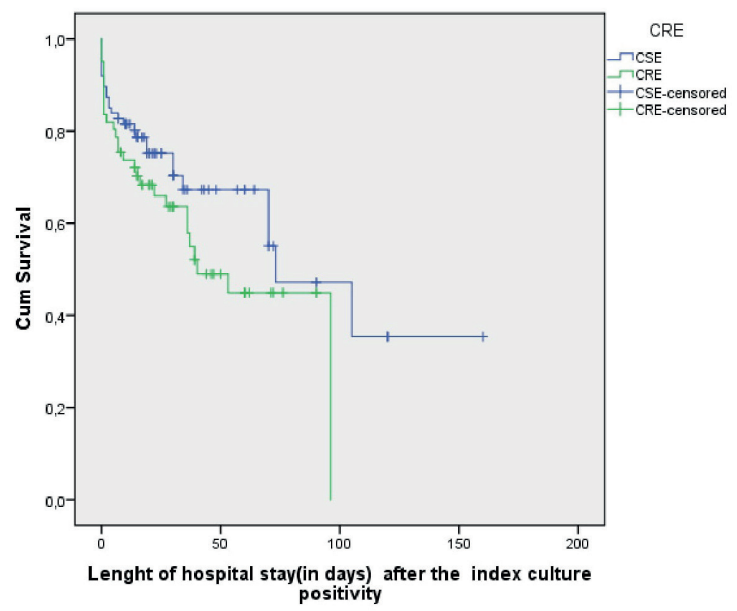

Fig. 1. Kaplan-Maier analysis comparing hospital survival of the patients with and without carbapenem resistance. (CRE: carbapenem-resistant Enterobactericeae, CSE: carbapenem-sensitive Enterobactericeae).

in in-hospital mortality rates between the colistin-susceptible and colistin-resistant Enterobacteriaceae infections $(\mathrm{p}=0.9)$. The median length of hospital stay after the index culture in colistin-resistant group was 20 days (range, 0 - 76 days) vs. 19 days (range, 0 - 160 days) in colistin-susceptible group $(\mathrm{p}=0.80)$.

\section{Discussion}

Our 4-year surveillance clearly confirmed that infections caused by Enterobacteriaceae isolates are an important clinical problem and high rates of carbapenem and/or colistin resistance can be encountered with these infections. We also found a high rate of colistin resistance that emerged over a period of four years.

The inability to recognize CRE infections when they first occur in a health care facility leads to the loss of the chance of early treatment choices before these infections are transmitted more widely. For this reason it is important to be aware of the risk factors for CRE infections. ${ }^{6}$ In our study empiric polyantibiotic therapy for Gram negative bacteria, long hospital stay before index culture and history of surgical intervention were found as risk factors of CRE infections. Mechanical ventilation prior to index culture, urinary catheter exposure, underlying necrotizing enterocolitis and/or short-bowel syndrome was found as risk factors of colistin resistance.

One of the most concerning risk factors of CRE infections found in our study was the empiric polyantibiotic therapy for Gram negative bacteria. Use of antimicrobials including carbapenems, cephalosporins, and fluoroquinolones associated with CRE infections or carriage of CRE has been reported in the literature. ${ }^{12-14}$

In a 4-year case-control study involving 102 patients, the only common variable associated with CRE infections was the cumulative number of antibiotic exposures prior to CRE infections. ${ }^{8}$ In another case-control study from Greece both prior exposure to antibiotics and duration of the prior antibiotic treatment were identified as the risk factors of CRE. ${ }^{15}$

Injudicious use of broad-spectrum agents may lead to the development of clinical resistance during therapy. ${ }^{16}$ Broad spectrum antibiotics can destroy the sensitive flora, and lead to the colonization and proliferation of the resistant mutant strains. ${ }^{16}$

There is an evolving body of medical literature suggesting an important relationship between prior antimicrobial therapy and the subsequent identification of carbapenemase-producing bacteria. Our data was consistent with previous reports that empiric polyantibiotherapy which was mentioned in our study carbapenem plus aminoglycoside and aminoglycoside combination therapy other than carbapenem were consistent with higher rates of CRE infections. In order to get rid of the "broadspectrum is best" approaches, we have to find novel ways to detect pathogens early.

Regarding the other risk factors other than antibiotic exposure, Patel et al. ${ }^{8}$ found that invasive infections with carbapenem-resistant K. pneumoniae were independently associated with longer length of stay when compared with patients with carbapenem-susceptible $K$. 
pneumoniae. Also in our study long hospital stay before index culture was also found as a risk factor of CRE. During long hospital stay patients can be exposed to multiple invasive procedures.

Surgical intervention particularly gastrointestinal procedures were found as highrisk procedures for CRE infections. ${ }^{17,18}$ Since most, ESBL-producing Enterobacteriaceae reside in the gastrointestinal tract it was not surprising to find this relationship. Although this relationship raises questions such as whether patients should be screened for ESBL-producing Enterobacteriaceae prior to surgery or whether modified surgical antibiotic prophylaxis in areas with high ESBL-producing Enterobacteriaceae prevalence is needed. ${ }^{19}$ However this topic was beyond the scope of our study. Also there is no known answer for these situations. Invasive CRE infections are associated with worse outcomes as compared to CSE infections. CRE isolation has been associated with all-cause hospital mortality ranging from $29 \%$ to $52 \%{ }^{20}$

In our study the rate was $45.2 \%$. Although CRE infections are known to be associated with high mortality, we did not find any difference between the mortality rates of CRE and CSE groups. Also in the study by Bhargava et al. ${ }^{21}$ and Candevir Ulu et al. ${ }^{22}$ mortality was not statistically different between carbapenemresistant and susceptiblestrains, which was similar to our study.

In the present study, the ratio of colistin-resistant infections in Enterobacteriaceae infections (32.3\%) was similar to what was previous reported by Zarkotou et al..$^{23}$ and Capone et al. ${ }^{3}$ which demonstrated $25 \%$ to $37 \%$ of resistance to colistin.

Although, colistin resistance has been associated with high mortality, there is controversy about the impact of resistance to colistin on prognosis. ${ }^{3,5,23-26}$ In our study mortality rates with colistin-resistant Enterobacteriaceae infections was not different from colistinsusceptible Enterobacteriaceae infections which was $45.2 \%$ and $45 \%$ respectively.
Our study has several limitations. Firstly, clinical data in this study were the single center experiences obtained from medical records of the patients retrospectively. Secondly, we used Vitex 2 for colistin MIC determination. Although, recently, a joint recommendation by CLSI and EUCAST released in 2016 recommended broth microdilution as a standard method for MIC testing of colistin, the use of broth microdilution methods for susceptibility may not be practical in laboratories depending on individual workloads and is rarely used in routine microbiology laboratories. ${ }^{27}$ Also Vitex 2 was previously reported as a good testing method for colistin MIC determination..$^{28,29}$ In the literature there are evolving studies evaluating the commercial testing methods comparing to broth microdilution for colistin MIC determination.

Further investigations will increase our understanding of these serious infections and give us an opportunity to find practices for reducing the frequency and the mortality of these infections.

\section{REFERENCES}

1. Ben-Ami R, Rodríguez-Baño J, Arslan H, et al. A multinational survey of risk factors for infection with extended-spectrum beta-lactamase-producing enterobacteriaceae in nonhospitalized patients. Clin Infect Dis 2009; 49: 682-690.

2. Temkin E, Adler A, Lerner A, Carmeli Y. Carbapenem-resistant Enterobacteriaceae: biology, epidemiology and management. Ann NY Acad Sci 2014; 1323: 22-42.

3. Capone A, Giannella M, Fortini D, et al. High rate of colistin resistance among patients with carbapenemresistant Klebsiella pneumoniae infection accounts for an excess of mortality. Clin Microbiol Infect 2013; 19: E23-E30.

4. Meletis G, Tzampaz E, Sianou E, Tzavaras I, Sofianou D. Colistin heteroresistance in carbapenemaseproducing Klebsiella pneumoniae. J Antimicrob Chemother 2011; 66: 946-947.

5. Kontopidou F, Plachouras D, Papadomichelakis E, et al. Colonization and infection by colistin-resistant gram-negative bacteria in a cohort of critically ill patients. Clin Microbiol Infec 2011; 17: E9-E11. 
6. Gupta N, Limbago BM, Patel JB, Kallen AJ. Carbapenem-resistant enterobacteriaceae: epidemiology and prevention. Clin Infect Dis 2011; 53: 60-67.

7. Ling ML, Tee YM, Tan SG, et al. Risk factors for acquisition of carbapenem resistant Enterobacteriaceae in an acute tertiary care hospital in Singapore. Antimicrob Resist Infect Control 2015; 4: 26 .

8. Patel N, Harrington S, Dihmess A, et al. Clinical epidemiology of carbapenem-intermediate or-resistant Enterobacteriaceae. J Antimicrob Chemother 2011; 66: 1600-1608.

9. Carbapenem-Resistant Enterobacteriaceae (CRE) Control and Prevention Toolkit 2012 CER Toolkit Atlanta, GA: CDC, 2012. Available at: www.cdc.gov/ hai/pdfs/cre/CRE-guidance-508.pdf [Updated 2015 November, cited 2017 May 15].

10. European Society of Clinical Microbiology and Infectious Diseases. European Committee on Antimicrobial Susceptibility Testing. Clinical breakpoints-bacteria(v7.1) [internet]. Switzerland: ESCMID, 2013. Available at: http:// www.eucast.org/ clinical_breakpoints/ [Updated 2017 Dec 31, cited 2018 May 15].

11. Horan TC, Andrus M, Dudeck MA. CDC/NHSN surveillance definition of health care-associated infection and criteria for specific types of infections in the acute care setting. Am J Infect Control 2008; 36: 309-332.

12. Patel G, Huprikar S, Factor SH, Jenkins SG, Calfee DP. Outcomes of carbapenem-resistant Klebsiella pneumoniae infection and the impact of antimicrobial and adjunctive therapies. Infect Control Hosp Epidemiol 2008; 29:1099-1106.

13. Hussein K, Sprecher H, Mashiach T, Oren I, Kassis I, Finkelstein R. Carbapenem resistance among Klebsiella pneumoniae isolates: risk factors, molecular characteristics, and susceptibility patterns. Infect Control Hosp Epidemiol 2009; 30: 666-671.

14. Schwaber MJ, Klarfeld-Lidji S, Navon-Venezia S, Schwartz D, Leavitt A, Carmeli Y. Predictors of carbapenem-resistant Klebsiella pneumoniae acquisition among hospitalized adults and effect of acquisition on mortality. Antimicrob Agents Chemother 2008; 52: 1028-1033.
15. Kritsotakis EI, Tsioutis C, Roumbelaki M, Christidou A, Gikas A. Antibiotic use and the risk of carbapenem-resistant extended-spectrum$\beta$-lactamase-producing Klebsiella pneumoniae infection in hospitalized patients: results of a double case-control study. J Antimicrob Chemother 2011; 66: 1383-1391.

16. Bell BG, Schellevis F, Stobberingh E, Goossens H, Pringle M. A systematic review and meta-analysis of the effects of antibiotic consumption on antibiotic resistance. BMC Infect Dis 2014; 14: 13.

17. Kofteridis DP, Valachis A, Dimopoulou D, et al Risk factors for carbapenem-resistant Klebsiella pneumoniae infection/colonization: a case-casecontrol study. J Infect Chemother 2014; 20: 293-297.

18. Debby BD, Ganor O, Yasmin M, et al. Epidemiology of carbapenem resistant Klebsiella pneumoniae colonization in an intensive care unit. Eur J Clin Microbiol Infect Dis 2012; 31: 1811-1817.

19. World Health Organization (WHO). Global Guidelines on the Prevention of Surgical Site Infection. Geneva: WHO, 2016. Available at: www. who.int/gpsc/appendix4.pdf. [Updated Nov 2016; May 2018].

20. Hyle EP, Ferraro MJ, Silver M, Lee H, Hooper DC. Ertapenem-resistant Enterobacteriaceae: risk factors for acquisition and outcomes. Infect Control Hosp Epidemiol 2010; 31: 1242-1249.

21. Bhargava A, Hayakawa K, Silverman E, et al. Risk factors for colonization due to carbapenem-resistant Enterobacteriaceae among patients exposed to long-term acute care and acute care facilities. Infect Control Hosp Epidemiol 2014; 35: 398-405.

22. Candevir Ulu A, Kurtaran B, Inal AS, et al. Risk factors of carbapenem-resistant Klebsiella pneumoniae infection: a serious threat in ICUs. Med Sci Monit2015; 21: 219-224.

23. Zarkotou O, Pournaras S, Tselioti P, et al. Predictors of mortality in patients with bloodstream infections caused by KPC-producing Klebsiella pneumoniae and impact of appropriate antimicrobial treatment. Clin Microbiol Infect 2011; 17: 1798--1803.

24. Humphries RM, Keledesis T, Dien Bard J, Ward KW, Bhattacharya D, Lewinski MA. Successful treatment of pan-resistant Klebsiella pneumoniae pneumonia and bacteraemia with a combination of high-dose tigecycline and colistin. J Med Microbiol 2010; 59: 1383-1386. 
25. van Duin D, Kaye KS, Neuner EA, Bonomo RA. Carbapenem-resistant Enterobacteriaceae: a review of treatment and outcomes. Diagn Microbiol Infect Dis 2013; 75: 115-120.

26. de Maio Carrilho CMD, de Oliveira LM, Gaudereto $\mathrm{J}$, et al. A prospective study of treatment of carbapenem-resistant Enterobacteriaceae infections and risk factors associated with outcome. BMC Infect Dis 2016; 16: 629.

27. European Society of Clinical Microbiology and Infectious Diseases. European Committee on Antimicrobial Susceptibility Testing (EUCAST). Recommendations for colistin (polymixin E) MIC testing-joint EUCAST and CLSI recommendation. internet]. Switzerland: ESCMID, 2016. Available at: http://www.eucast.org/ast_of_bacteria/guidance_ documents/ [Updated 2016 March 22, cited 2018 May 5].
28. Dafopoulou K, Zarkotou O, Dimitroulia E, et al. Comparative evaluation of colistin susceptibility testing methods among carbapenem-nonsusceptible Klebsiella pneumoniae and Acinetobacter baumannii clinical isolates. Antimicrob Agents Chemother 2015; 59: 4625-4630.

29. Lee SY, Shin JH, Lee K, et al. Comparison of the vitex 2, microsan and etest methods with the agar dilution method in assessing colistin susceptibility of bloodstream isolates of Acinetobacter species from a Korean University Hospital. J Clin Microbiol 2013; 51: 1924-1926. 